\title{
Sonographic Hepatorenal Ratio: A Noninvasive Method to Diagnose Nonalcoholic Steatosis
}

\author{
Valéria Ferreira de Almeida e Borges, $\mathrm{MD},{ }^{1}$ Angélica L. D. Diniz, PhD, ${ }^{1}$ \\ Helma P. Cotrim, PhD, ${ }^{2}$ Haroldo L. O. G. Rocha, MD, ${ }^{1}$ Nestor Barbosa Andrade, MD $^{1}$ \\ ${ }^{1}$ Universidade Federal de Uberlândia, Uberlândia, Minas Gerais, Brazil \\ ${ }^{2}$ Universidade Federal da Bahia, Salvador, Bahia, Brazil
}

Received 13 May 2011; accepted 13 August 2012

\begin{abstract}
Purpose. To evaluate the accuracy of the sonographic hepatorenal ratio (HRR) in the diagnosis and grading of nonalcoholic steatosis, using biopsy as the reference.

Methods. Ultrasound (US) and liver biopsy were performed in 42 patients with nonalcoholic fatty liver disease. Forty healthy volunteers without steatosis at US and without risk factors for nonalcoholic fatty liver disease were also studied. The HRR was obtained by dividing the mean brightness level of region-of-interest pixels in hepatic parenchyma by that in renal parenchyma. Needle biopsy samples (hematoxylin-eosin stained) were classified as mild (5-33\% fatty infiltration), moderate $(>33-66 \%)$, or severe $(>66 \%)$ steatosis. Spearman coefficient was used to evaluate the correlation between HRR and steatosis grade, analysis of variance for differences between subgroups, and receiver operating characteristic curve analysis for sensitivity and specificity.

Results. Significant correlation was found between HRR and histologic steatosis $(r=0.80, p<0.01)$. The HRR cutoff for predicting steatosis was $\geq 1.24$ (sensitivity, 92.7\%; specificity, 92.5\%). The mean \pm SD HRRs in controls and steatosis subgroups were control $1.09 \pm 0.13$, mild $1.46 \pm 0.24$, moderate $1.52 \pm$ 0.27 , severe $2.04 \pm 0.3$ and were significantly different from each other except between mild and moderate steatosis subgroups.

Conclusions. The HRR is a noninvasive, objective, and simple method that could be used to diagnose and grade hepatic steatosis. () 2012 Wiley Periodicals, Inc. J Clin Ultrasound 41:18-25, 2013; Published online in Wiley Online Library (wileyonlinelibrary. com). DOI: 10.1002/jcu.21994
\end{abstract}

Correspondence to: V. Ferreira de Almeida e Borges

(C) 2012 Wiley Periodicals, Inc.
Keywords: fatty liver; ultrasound; sonographic hepatorenal ratio; liver biopsy; nonalcoholic steatosis

$\mathrm{N}$ onalcoholic fatty liver disease (NAFLD), a common cause of chronic liver disease and largely associated with the current epidemics of obesity and diabetes, refers to a spectrum of liver damages ranging from simple steatosis to steatohepatitis and subsequently fibrosis, and progression to cirrhosis and liver cancer. ${ }^{1,2}$ NAFLD is usually found incidentally after recognition of a mild elevation of aminotransferase levels or steatosis on routine clinical interpretation of ultrasound (US) performed for other purposes, and increasingly it is recognized as the hepatic manifestation of metabolic syndrome. ${ }^{3-6}$

The "gold standard" method to diagnose steatosis is the histology of a liver sample collected by biopsy, a procedure that is both invasive and painful and presents some risks. ${ }^{7,8}$ In addition, the very small liver sample may not be representative of the whole liver, especially in cases of heterogeneous fat distribution. Besides being subject to significant sampling variability, another limitation of biopsy is that a pathologist normally performs an estimation of fat semiquantitatively. ${ }^{9}$

Although blood analyses provide markers of steatosis, so far, establishing reliable correlations with histologic results has not been possible. ${ }^{10}$

Noninvasive diagnostic imaging methods have been applied to steatosis detection. For example, US, which is widely used to detect hepatic steatosis, has the advantage of being safe, inexpensive, 
and readily available; however, US is operatordependent and its sensitivity is reduced in the morbidly obese as well as in those with small amounts of fatty infiltration. ${ }^{11,12}$ Another imaging method for detecting steatosis is CT, but this technique exposes subjects to ionizing radiation, thus limiting its use in longitudinal studies and in children. ${ }^{13}$ Both MRI and spectroscopy have been investigated in the last few years for the detection of steatosis but are relatively expensive and involve risks related to the contrast materials, as well as claustrophobic reactions, which are limitations for this imaging method. ${ }^{14,15}$

Prior studies have analyzed the histogram of the sonographic level of brightness (a graphic representation of echo intensity) in an attempt to grade steatosis and in seeking to solve US subjectivity. ${ }^{16-18}$ However, the methodological characteristics of these studies, such as absence of a histologic reference, retrospective design, or heterogeneous study group comprising patients with chronic liver diseases of different causes, could compromise a conclusion about the data published. Furthermore, most studies comparing imaging methods with liver biopsy included patients with severity predictors, such as elevated liver enzyme levels, thrombocytopenia, morbid obesity, and age older than 45 years, which can also lead to bias. ${ }^{19}$

Webb et al ${ }^{18}$ studied 111 patients with chronic hepatopathy (from different causes) who underwent liver biopsy; these authors observed that the sonographic hepatorenal ratio (HRR) using histograms can quantify the grade of steatosis accurately. Osawa and Mori ${ }^{17}$ using CT as the reference, observed that the HRR was able to predict steatosis with $91.3 \%$ sensitivity, $83.8 \%$ specificity, and $86.7 \%$ accuracy. Mancini et al ${ }^{16}$ used MR as the reference standard when they studied the HRR in obese individuals and those with diabetes.

The need for an accurate noninvasive method to diagnose steatosis is especially important for the following reasons: steatosis is becoming widespread in the pediatric population, in whom invasive methods must be avoided ${ }^{20,21}$; liver steatosis can increase the risk of postsurgical complications after partial hepatic resection ${ }^{22}$ in the preoperative evaluation of living-donor livers; steatosis places the graft at risk for dysfunction and can negatively affect the outcome of hepatic transplantation ${ }^{23}$; among all liver lesions, steatosis seems to be the one most remarkably affected by therapy for $\mathrm{NAFLD}^{24}$; clinically, fatty liver is an important sign in predicting the risk of diabetes and cardiovascular accidents. ${ }^{25}$
Given the high prevalence and clinical importance of fatty liver as well as the fact that a positive diagnosis of fatty liver is commonplace in the routine clinical interpretation of US, which is widely used often without concomitant histogram, we believe it is important to understand the capability of US in diagnosing steatosis and to develop an approach to diagnosing and classifying fatty liver with a greater consistency, rather than disregarding it. We, therefore, developed this work because of the enormous prevalence of NAFLD in clinical practice, with different US descriptions of the level of steatosis.

The purpose of this study was to prospectively evaluate the accuracy of the parameter HRR when diagnosing and grading steatosis in patients with NAFLD, using needle biopsy of the liver as the reference standard.

\section{MATERIALS AND METHODS}

This was an observational study, with prospective data collection, performed at a single research center, during the period between October 2008 and November 2009. The volunteers were included consecutively. The research protocol was approved by the local Ethics in Research Committee (Registration number 065/08) and only volunteers who signed the informed consent form after hearing a verbal explanation were included.

Inclusion criteria were patients aged 18-70 years, of either sex, with the presence of steatosis verified by conventional US according to established criteria. ${ }^{26}$ Forty-two patients were included in the steatosis group. Forty healthy volunteers without steatosis at US and without risk factors for NAFLD were also studied. This group was chosen based on lifestyle, no risk factors for NAFLD, no or minimum alcohol intake, no diabetes or obesity, and no characteristic sign of insulin resistance.

Exclusion criteria were as follows: significant alcohol intake $>140 \mathrm{~g} /$ week for men and $>70 \mathrm{~g} /$ week for women ${ }^{27}$; viral hepatitis $\mathrm{C}$ or $\mathrm{B}^{28}$; other liver diseases; portal hypertension, ascitis, liver failure; blood coagulation disorder; cardiac diseases or coronary, cerebral, or peripheral acute ischemia; respiratory failure; focal steatosis; nephropathy (based on serum urea and creatinine levels, urine sediment, and renal US); ectopic or absent right kidney; focal liver lesions; focal kidney lesions; pregnancy or lactation.

The healthy volunteers were submitted to the same clinical, laboratory, and US evaluations as the patient group except for liver biopsy, for ethical reasons. All patients in the steatosis 
group followed the inclusion and exclusion criteria described above. The volunteers group followed the same criteria, except for the presence of liver steatosis at US. The age of the volunteers at the time of their selection was the same when the US and the biopsy were done.

Insulin resistance was calculated by means of the homeostasis model assessment as follows: [serum insulin $(\mathrm{IU} / \mathrm{ml}) \times$ fasting glucose $(\mathrm{mg} / \mathrm{dl})] /$ $405 .^{29,30}$ Patients who were diabetic or prediabetic were considered insulin resistant.

All 82 participants were examined by US in B-mode, using a 2- to 5-MHz multifrequency convex transducer (Voluson 730 PRO V; General Electric, Milwaukee, WI), in a fasting state, in the dorsal decubitus position, with the arms elevated. The same operator, with 20 years of experience, performed all US examinations.

All segments of the liver and kidneys were examined, and patients with findings of vascular malformations, cysts, and focal parenchymal lesions were excluded. Those patients with renal changes, atrophy, or ectopy of the right kidney were also excluded.

The steatosis diagnosis was based on grayscale US findings of fatty infiltration of the liver, which included diffuse increase of echogenicity, increased acoustic attenuation, and masking of the diaphragm and vessel walls. ${ }^{26}$

Histograms were used to obtain a numerical measure of echo intensity. To acquire them, the probe was placed in a right subcostal coronal position on each volunteer until stable images of the liver and renal parenchyma could be obtained. Regions of interest (ROIs), approximately $1.2 \times 1.2 \mathrm{~cm}$, were selected so as to contain only hepatic and renal parenchyma, without any visible vessel, renal sinus, or medulla. The hepatic and renal ROIs were selected on the same plane and depth to avoid image distortion effects. The ROIs' parameters displayed were mean brightness level and SD. The HRR was obtained by dividing the mean brightness level of ROI pixels in hepatic parenchyma by those in renal parenchyma (Figure 1). The blue charts in Figure 1 have the brightness level in the abscissas and pixel's number in the ordinates.

A US-guided Tru-Cut needle was used to perform a biopsy of the right lobe of the liver. The liver fragment, about $10 \mathrm{~mm}$, was fixed in formalin $4 \%$ and staining was performed with hematoxylin-eosin and Masson's Trichrome. A single experienced pathologist evaluated the material, blind to the corresponding US data. The biopsy was performed at a maximum interval of 1 week after or before the US examination.

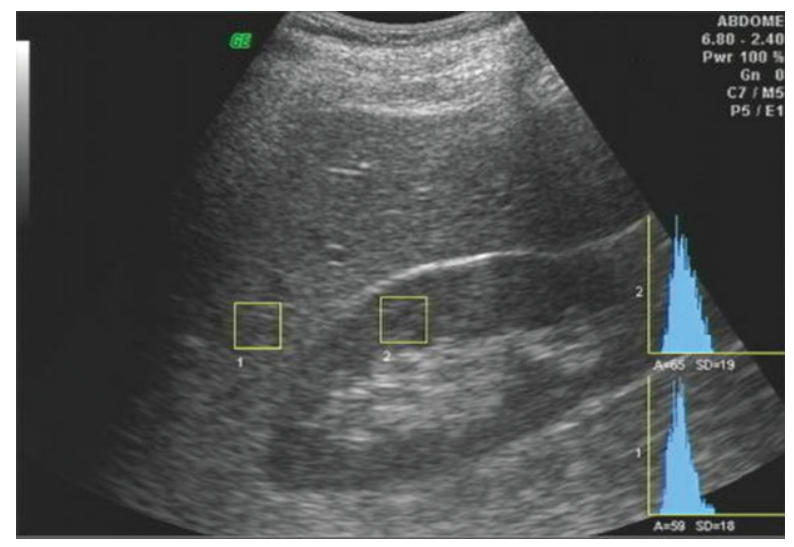

FIGURE 1. Coronal sonogram shows the $1.2 \times 1.2 \mathrm{~cm}$ regions of interest (boxes) located in the hepatic (\#1) and renal parenchyma (\#2) and the respective histograms. The charts show the brightness level in the abscissa and pixel's numbers in the ordinate.

The histologic evaluation was based on the standardized classification of the pathology committee review of the Nonalcoholic Steatohepatitis (NASH) Clinical Research Network. ${ }^{31}$

The histologic variables were described and subdivided into hepatocellular alterations, fibrosis, and inflammatory infiltrate. The following hepatocellular alterations were systematically sought: steatosis, hepatocellular balloonization, and Mallory's hyaline corpuscle. Steatosis was classified as mild $(<33 \%$ of the hepatocytes infiltrated by fat droplets), moderate (33\% to 66\%), or severe $(>66 \%)$. Inflammatory infiltrate was discriminated as mild, moderate, or severe intensity. Fibrosis was described according to location in the hepatic acinus, aspect, and intensity.

Statistical analysis was performed by using the Statistical Package for Social Sciences software (SPSS for Windows, version 16.0, Chicago, IL). The Spearman coefficient was used to evaluate the correlation between HRR and histologic grade of steatosis. One-way analysis of variance was used to evaluate group differences, and Tukey's test was applied for post-hoc analysis. Sensitivity and specificity were calculated by using receiver operating characteristic (ROC) curve analysis. The categorical variables were expressed by absolute (n) and relative frequency (\%) and analyzed with the Mann--Whitney and Fisher tests.

All significance tests were two-sided, and a level of significance of $0.05(\alpha=5 \%)$ was adopted; descriptive levels ( $p$ values) lower than this value were considered significant.

\section{RESULTS}

The mean age was $42 \pm 12$ years (range, 27-70 years) in the volunteers group and $47 \pm 10$ years 
TABLE 1

Clinical and Laboratory Characteristics of the Steatosis Group and Control Group

\begin{tabular}{|c|c|c|c|}
\hline Characteristic & $\begin{array}{l}\text { Steatosis } \\
(n=42)\end{array}$ & $\begin{array}{c}\text { Control } \\
(\mathrm{n}=40)\end{array}$ & $p$ Value \\
\hline Male, n (\%) & $7(17)$ & $11(28)$ & 0.291 \\
\hline Age (years) & $47 \pm 10$ & $42 \pm 12$ & 0.090 \\
\hline Albumin (g/dL) & $4.7 \pm 0.52$ & $4.7 \pm 0.41$ & 0.955 \\
\hline Platelets $/ 1,000$ & $276 \pm 75$ & $256 \pm 49$ & 0.146 \\
\hline Transferrin saturation index & $30 \pm 13$ & $32 \pm 11$ & 0.360 \\
\hline Total bilirubin (mg/dL) & $0.64 \pm 0.57$ & $0.79 \pm 0.42$ & 0.206 \\
\hline \multicolumn{4}{|l|}{ Waist circumference $(\mathrm{cm})$} \\
\hline Men & $4 \pm$ & $85 \pm 11$ & $<0.001$ \\
\hline Women & $103 \pm 11$ & $75 \pm 7$ & $<0.001$ \\
\hline BMI $\left(\mathrm{kg} / \mathrm{m}^{2}\right)$ & $32 \pm 6$ & $22 \pm 2$ & $<0.001$ \\
\hline $\mathrm{mg} / \mathrm{dl})$ & $105 \pm 23$ & $85 \pm 7$ & $<0.001$ \\
\hline HOMA-IR & $2.84 \pm 2.77$ & $0.88 \pm 0.49$ & 0.001 \\
\hline Total chole & $198 \pm 36$ & $180 \pm 40$ & 0.049 \\
\hline Triglycerides & $163 \pm 82$ & $89 \pm 41$ & $<0.001$ \\
\hline $\mathrm{HDL}(\mathrm{mg} / \mathrm{dl})$ & $45 \pm 10$ & $60 \pm 11$ & $<0.001$ \\
\hline Aspartate aminotransferase ${ }^{*}$ & $0.79 \pm 0.65$ & $0.38 \pm 0.11$ & $<0.001$ \\
\hline Alanine aminotransferase ${ }^{*}$ & $1.08 \pm 0.79$ & $0.41 \pm 0.18$ & $<0.001$ \\
\hline Gamma-glutamyl transferas & $1.49 \pm 1.29$ & $0.46 \pm 0.23$ & $<0.001$ \\
\hline Alkaline phosphatase ${ }^{*}$ & $0.75 \pm 0.28$ & $0.53 \pm 0.16$ & $<0.001$ \\
\hline Ferritin (ng/mL) & $207 \pm 131$ & $123 \pm 135$ & 0.007 \\
\hline
\end{tabular}

Abbreviations: BMI, body mass index; HOMA-IR, homeostasis model assessment for insulin resistance; HDL, high-density lipoprotein cholesterol; ns, not significant.

Except for male, data are mean \pm SD.

* The liver enzymes are described in relation to the respective upper limit of normal.

in the steatosis group. Using one-way analysis of variance, no significant difference was found between the mean ages $(p=0.09)$. The volunteers consisted of 11 men and 29 women, and the steatosis group included 7 men and 35 women. No significant difference was seen between these proportions $(p=0.29)$ using the Fisher test. Also, no significant difference was found between the groups concerning serum albumin level, bilirubin, number of platelets, and transferrin saturation index. However, the patients with NAFLD had elevated rates of obesity, dyslipidemia, insulin resistance, liver enzymes, and ferritin levels compared with the controls (Table 1).

Biopsy results confirmed the presence of steatosis in all 42 patients with US-diagnosed steatosis. Per histologic evaluation, 17 patients had mild steatosis, 20 patients had moderate steatosis, and 5 patients had severe steatosis.

All patients with steatosis met NASH criteria, and no case of pure steatosis was found. Lobular inflammation was mild in 30 patients (71\%), moderate in $4(10 \%)$, and marked in $2(5 \%)$. Portal inflammation was absent to minimal in 3 patients $(7 \%)$ and greater than minimal in 39 patients $(93 \%)$. Ballooning degeneration was identified in 39 (93\%) of the 42 patients of the steatosis group, 25 of whom had many ballooned cells. No Mallory bodies were found using routine staining.
TABLE 2

Sonographic Hepatorenal Ratio According to the Histological Grade of Steatosis

\begin{tabular}{lccc}
\hline & & \multicolumn{2}{c}{$\begin{array}{c}\text { Sonographic } \\
\text { Hepatorenal Ratio }\end{array}$} \\
\cline { 3 - 4 } Group & Mean & $\begin{array}{c}\text { Standard } \\
\text { Deviation }\end{array}$ & $\begin{array}{c}95 \% \text { Confidence } \\
\text { Interval }\end{array}$ \\
\hline $\begin{array}{l}\text { Control }(\mathrm{n}=40) \\
\text { Steatosis subgroup }\end{array}$ & 1.09 & 0.13 & $1.05-1.13$ \\
$\quad \begin{array}{l}\text { Mild }(\mathrm{n}=17) \\
\quad \text { Moderate }(\mathrm{n}=20)\end{array}$ & 1.46 & 0.24 & $1.33-1.58$ \\
$\quad$ Severe $(\mathrm{n}=5)$ & 2.04 & 0.27 & $1.40-1.65$ \\
\hline
\end{tabular}

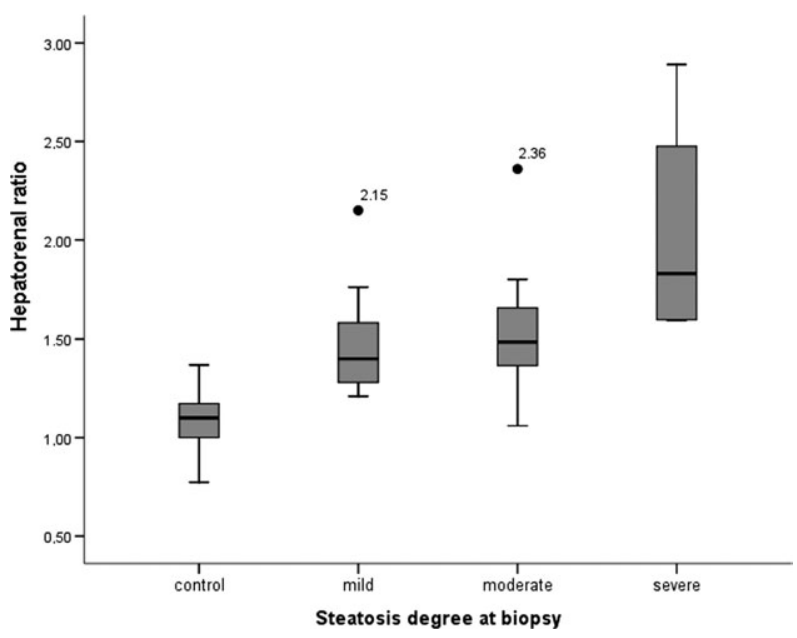

FIGURE 2. Boxplot graph of the sonographic hepatorenal ratio according to the histologic grade of steatosis. The horizontal line dividing each box is the median. The values refer to the outliers.

None of the patients evaluated had advanced fibrosis that would produce nodulations (cirrhosis) at biopsy. Thirty patients $(71 \%)$ had some degree of fibrosis, and bridging fibrosis was described in 9 (21\%).

The HRR positively correlated with the grade of steatosis at biopsy. As liver echogenicity increased in relation to the renal parenchyma, an increase in HRR proportional to the increased fatty infiltration at liver biopsy was noted. A direct and significantly close correlation was observed between HRR and the grade of steatosis at biopsy $(r=0.80, p<0.01)$, inflammation $(r=$ $0.71, p<0.05)$, and fibrosis $(r=0.58, p<0.05)$.

The mean HRR in the volunteers group was $1.09 \pm 0.13$ and in the steatosis group overall was $1.55 \pm 0.33(p<0.01)$. The ratios for the steatosis subgroups appear in Table 2 and were found to be significantly different from each other, except between the mild and moderate steatosis subgroups. Figure 2 shows the medians of the HRRs for all groups. Outliers are present in the mild and moderate steatosis groups. 
A ROC curve and two-graph ROC were applied to define the optimal HRR cutoff point for the diagnosis of fatty liver on basis of liver biopsy. The optimal cutoff point of HRR to diagnose steatosis was $\geq 1.24$ with $92.7 \%$ sensitivity and $92.5 \%$ specificity. Graphically, this corresponds to the point of the ROC curve closest to the upper left of the graph (point 0,1 ), ie, closer to the point on the graph whose sensitivity $=100 \%$ and specificity $=100 \%$ (Figure 3). It identifies the cutoff point that determines the highest sensitivity and specificity together (ie, for the same period). Considering the prevalence in the general population most often cited in the literature, which is $30 \%$, a positive predictive value of $84.1 \%$, negative predictive value of $96.7 \%$, and test accuracy of $92.6 \%$ were calculated.

According to the ROC curve analysis of the HRR used to diagnose steatosis, the area under the curve was $96.4 \%$ (95\% confidence interval: 92.5-100\%) (Figure 3).

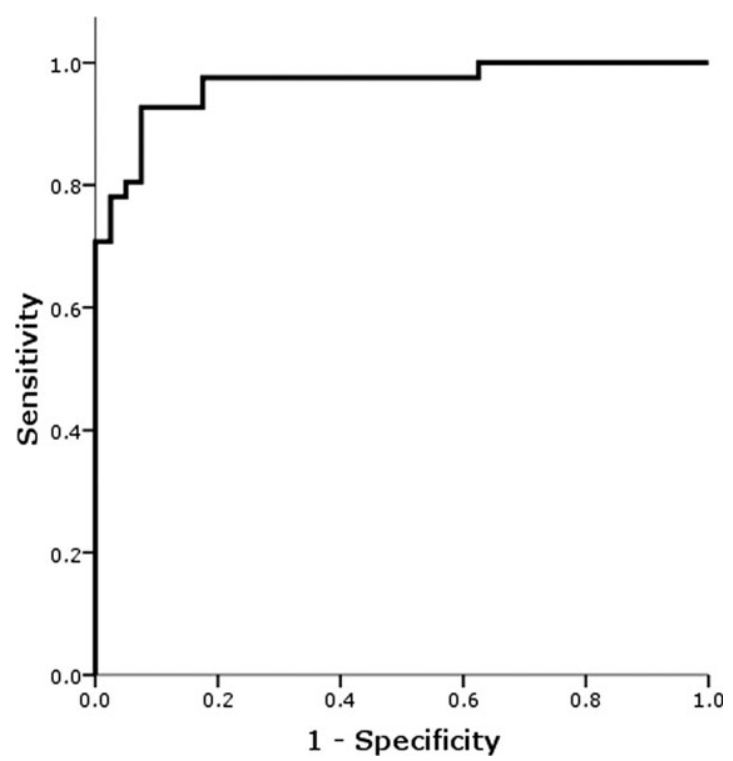

FIGURE 3. Graph of receiver operating characteristic curve of sensitivity plotted against 1 - specificity of sonographic hepatorenal ratio to diagnose steatosis seen at biopsy.

\section{DISCUSSION}

NAFLD is a common cause of chronic liver disease in individuals with insulin resistance. ${ }^{5,29,32}$ Its spectrum of manifestation begins with steatosis and can develop into steatohepatitis, fibrosis, cirrhosis, and hepatocellular carcinoma. ${ }^{2,33}$ The most frequent manifestation is the finding that suggests steatosis at US. ${ }^{34}$

To assess the degree of hepatic fat content, simple and noninvasive methods with high objectivity and reproducibility are required. The conventional US examination is very accurate for detecting moderate and severe steatosis, but the diagnosis of mild steatosis may be difficult without a computed imaging method. The echogenicity of the liver, compared subjectively with the kidney examination, is a clinically useful marker for steatosis.

This study examined the capacity of HRR to predict the degree of liver fat, and the data showed a good correlation between the HRR and the grade of steatosis, using biopsy as the reference standard method. The steatosis grade is related to prognosis, ${ }^{35}$ and it is also important in the assessment of the therapeutic response. ${ }^{24,36}$

In this study, the volunteers group had no risk factors for NAFLD and was confirmed to be healthy, without any characteristic sign of insulin resistance, which clearly distinguished them from the patients in the steatosis group. In the population studied here, it was demonstrated that the intensity of the mean brightness level of liver echoes, slightly higher than the renal one (HRR >1.24), defined the presence of liver steatosis by means of US, with a sensitivity of $92.7 \%$ and specificity of $92.5 \%$ compared with the histologic examination.

Prior studies observed a linear relationship between the presence of steatosis and the coefficient of hepatic attenuation. ${ }^{37-39}$ The results of these studies agree with those that proposed to use the difference between the mean brightness

TABLE 3

Main Studies Available in the Literature That Used the Sonographic Hepatorenal Ratio to Grade Liver Steatosis

\begin{tabular}{|c|c|c|c|c|c|c|c|}
\hline Study & Sample & $\mathrm{n}$ & $\begin{array}{l}\text { Reference } \\
\text { Standard }\end{array}$ & Design & Cutoff & Sens. (\%) & Specif (\%) \\
\hline Osawa, $1996^{17}$ & $\begin{array}{l}\text { Nonalcoholic and nonviral } \\
\text { liver steatosis }\end{array}$ & 70 & СТ & Prospective & --- & 91.3 & 83.8 \\
\hline Kim, $2005^{40}$ & Liver steatosis in liver donors & 94 & Biopsy* & Retrospective & --- & --- & ---- \\
\hline Webb, $2009^{18}$ & $\begin{array}{l}\text { Liver diseases due to } \\
\text { various causes }\end{array}$ & 111 & Biopsy* $^{*}$ & Retrospective & 1.49 & 100 & 91 \\
\hline Mancini, $2009^{16}$ & NAFLD & 40 & MRI & Prospective & 2.20 & 100 & 95 \\
\hline Current study & NAFLD & 82 & Biopsy $^{*}$ & Prospective & 1.24 & 93 & 93 \\
\hline
\end{tabular}

Abbreviations: NAFLD, nonalcoholic fatty liver disease; Sens, sensitivity; Specif, specificity.

* Reference standard for the patient group. 
level of the liver echo and the renal parenchyma as a way to overcome the variability of subjective evaluation. ${ }^{16-18,40}$

Table 3 shows the main characteristics of the studies available in the literature. ${ }^{16-18,40}$ The methodology is as close as possible to our current study, but our study is different from those cited because it is prospective, the reference is liver biopsy, and we evaluated fibrosis and inflammation. The population studied was homogeneous (only those with NAFLD). We also included volunteers because of the presence of steatosis at US (later confirmed by histology), not necessarily accompanied by high aminotransferase levels or by surrogates of severity. This inclusion criterion brings the sample studied closer to the general population of patients with NAFLD.

It was previously reported that US sensitivity and specificity to detect fatty infiltration of the liver diminish as body mass index increases. ${ }^{11}$ The sensitivity and specificity of US to diagnose steatosis in patients with morbid obesity were $64.9 \%$ and $90.9 \%$, respectively. ${ }^{11}$ This implies a limitation in the evaluation of populations with a high prevalence of obesity. As the attenuation of the beam caused by subcutaneous fat will be the same on the liver as on the renal parenchyma, the use of the HRR in obese patients is a reliable feature.

Liver fibrosis causes heterogeneity in the echotexture of the parenchyma and could, theoretically, make it difficult to evaluate steatosis. This issue of the interference of the parenchyma in the pattern of brightness was evaluated in two studies in $1986^{38,39}$ and more recently by Palmentieri et al, ${ }^{37}$ who showed that only steatosis was significantly correlated with the bright liver. In our study, there was also a higher correlation of HRR with the grade of steatosis than with fibrosis or inflammation. Thus, the bright liver standard may be considered a more specific clinical sign of steatosis than of fibrosis.

The steatosis group was chosen on the basis of conventional US findings. It is theoretically possible that patients whose results were considered normal at US had been excluded, although there was indeed fatty infiltration. This fact does not invalidate the method for steatosis diagnosis.

A limitation of the use of HRR is the presence of renal disease. Some severe renal diseases may increase parenchyma echogenicity, which would impair the comparison between liver and kidney, as would a right ectopic or absent kidney. ${ }^{41}$ In our study, nephropathy was excluded on the basis of the absence of risk factors, normal levels of urea and serum creatinine, normal kidneys at
US, and the absence of alterations in analyses of sediment. Another limitation is the heterogeneous distribution of fatty infiltration, as occurs in focal hepatic steatosis, resulting in a sampling error, independent of the methodology used, even with a biopsy. In the presence of focal steatosis or heterogeneous distribution, the measure of echo intensity in only a single ROI would not be representative of the entire liver and thus would compromise the quantification of steatosis. In these cases, results of the means of various areas in standardized locations could supply a representative quantification.

Only a single examiner compiled data referring to hepatic and renal intensity measurements. Reproducibility was not a goal of the present study.

Another limitation is the fact that the US operator was aware of the case or control status of the patients, which is a potential source of bias. However, the HRR is an objective parameter, with the potential to solve the US subjectivity. Besides, the methodology used was previously established ${ }^{26,31}$ and the US operator did not have access to the biopsy data.

\section{CONCLUSION}

The study showed that the accuracy of the sonographic HRR could be useful to diagnose and grade hepatic steatosis. There was a higher correlation with the grade of steatosis than with the grade of inflammation or grade of fibrosis at liver biopsy.

\section{REFERENCES}

1. Farrell GC, Larter CZ. Nonalcoholic fatty liver disease: from steatosis to cirrhosis. Hepatology 2006;43:S99.

2. Hashizume H, Sato K, Takagi H, et al. Primary liver cancers with nonalcoholic steatohepatitis. Eur J Gastroenterol Hepatol 2007;19:827.

3. Hamaguchi M, Kojima T, Itoh Y, et al. The severity of ultrasonographic findings in nonalcoholic fatty liver disease reflects the metabolic syndrome and visceral fat accumulation. Am J Gastroenterol 2007;102:2708.

4. Kirovski G, Schacherer D, Wobser H, et al. Prevalence of ultrasound-diagnosed non-alcoholic fatty liver disease in a hospital cohort and its association with anthropometric, biochemical and sonographic characteristics. Int $J$ Clin Exp Med 2010;3:202.

5. Machado M, Cortez-Pinto H. Non-alcoholic fatty liver disease and insulin resistance. Eur J Gastroenterol Hepatol 2005; 17:823. 
6. Narciso-Schiavon JL, Schiavon Lde L, CarvalhoFilho RJ, et al. Clinical characteristics associated with hepatic steatosis on ultrasonography in patients with elevated alanine aminotransferase. São Paulo Med J 2010;128:342.

7. Castera L, Negre I, Samii K, et al. Pain experienced during percutaneous liver biopsy. Hepatology 1999;30:1529.

8. Joy D, Thava VR, Scott BB. Diagnosis of fatty liver disease: is biopsy necessary? Eur J Gastroenterol Hepatol 2003;15:539.

9. Ratziu V, Charlotte F, Heurtier A, et al. Sampling variability of liver biopsy in nonalcoholic fatty liver disease. Gastroenterology 2005;128:1898.

10. Mofrad P, Contos MJ, Haque M, et al. Clinical and histologic spectrum of nonalcoholic fatty liver disease associated with normal ALT values. Hepatology 2003;37:1286.

11. de Moura Almeida A, Cotrim HP, Barbosa DB, et al. Fatty liver disease in severe obese patients: diagnostic value of abdominal ultrasound. World J Gastroenterol 2008;14:1415.

12. Strauss S, Gavish E, Gottlieb P, et al. Interobserver and intraobserver variability in the sonographic assessment of fatty liver. AJR Am J Roentgenol 2007;189:W320.

13. Lee SW, Park SH, Kim KW, et al. Unenhanced CT for assessment of macrovesicular hepatic steatosis in living liver donors: comparison of visual grading with liver attenuation index. Radiology 2007;244:479.

14. Cesbron-Metivier E, Roullier V, Boursier J, et al. Noninvasive liver steatosis quantification using MRI techniques combined with blood markers. Eur J Gastroenterol Hepatol 2010;22:973.

15. van Werven JR, Marsman HA, Nederveen AJ, et al. Assessment of hepatic steatosis in patients undergoing liver resection: comparison of US, CT, T1weighted dual-echo MR imaging, and point-resolved 1H MR spectroscopy. Radiology 2010;256:159.

16. Mancini M, Prinster A, Annuzzi G, et al. Sonographic hepatic-renal ratio as indicator of hepatic steatosis: comparison with (1)H magnetic resonance spectroscopy. Metabolism 2009;58:1724.

17. Osawa H, Mori Y. Sonographic diagnosis of fatty liver using a histogram technique that compares liver and renal cortical echo amplitudes. J Clin Ultrasound 1996;24:25.

18. Webb M, Yeshua H, Zelber-Sagi S, et al. Diagnostic value of a computerized hepatorenal index for sonographic quantification of liver steatosis. AJR Am J Roentgenol 2009;192:909.

19. Festi D, Colecchia A, Sacco T, et al. Hepatic steatosis in obese patients: clinical aspects and prognostic significance. Obes Rev 2004;5:27.

20. Loomba R, Sirlin CB, Schwimmer JB, et al. Advances in pediatric nonalcoholic fatty liver disease. Hepatology 2009;50:1282.

21. Pacifico L, Poggiogalle E, Cantisani V, et al. Pediatric nonalcoholic fatty liver disease: A clinical and laboratory challenge. World J Hepatol 2010;2:275.
22. Kooby DA, Fong Y, Suriawinata A, et al. Impact of steatosis on perioperative outcome following hepatic resection. J Gastrointest Surg 2003;7:1034.

23. Kim SH, Lee JM, Han JK, et al. Hepatic macrosteatosis: predicting appropriateness of liver donation by using MR imaging - correlation with histopathologic findings. Radiology 2006;240:116.

24. Belfort R, Harrison SA, Brown K, et al. A placebocontrolled trial of pioglitazone in subjects with nonalcoholic steatohepatitis. N Engl J Med 2006;355:2297.

25. Tarquini R, Lazzeri C, Boddi M, et al. [Non-alcoholic fatty liver disease: a new challenge for cardiologists.] G Ital Cardiol (Rome) 2010;11:660.

26. Charatcharoenwitthaya $\mathrm{P}$, Lindor $\mathrm{KD}$. Role of radiologic modalities in the management of nonalcoholic steatohepatitis. Clin Liver Dis 2007;11:37.

27. Farrell GC. An introduction to NASH and related fatty liver disorders. In: Farrell GC, George J, de la M Hall P, et al, editors. Fatty liver disease: NASH and related disorders. Malden: Blackwell Publishing; 2005, p 1.

28. Minakari M, Molaei M, Shalmani HM, et al. Liver steatosis in patients with chronic hepatitis B infection: host and viral risk factors. Eur J Gastroenterol Hepatol 2009;21:512.

29. Guidorizzi de Siqueira AC, Cotrim HP, Rocha R, et al. Non-alcoholic fatty liver disease and insulin resistance: importance of risk factors and histological spectrum. Eur J Gastroenterol Hepatol 2005;17:837.

30. Turner RC, Holman RR, Matthews D, et al. Insulin deficiency and insulin resistance interaction in diabetes: estimation of their relative contribution by feedback analysis from basal plasma insulin and glucose concentrations. Metabolism 1979;28:1086.

31. Kleiner DE, Brunt EM, Van Natta M, et al. Design and validation of a histological scoring system for nonalcoholic fatty liver disease. Hepatology 2005;41:1313.

32. Cusi K. Role of insulin resistance and lipotoxicity in non-alcoholic steatohepatitis. Clin Liver Dis 2009;13:545.

33. Charlton M. Cirrhosis and liver failure in nonalcoholic fatty liver disease: molehill or mountain? Hepatology 2008;47:1431.

34. Wieckowska A, Feldstein AE. Diagnosis of nonalcoholic fatty liver disease: invasive versus noninvasive. Semin Liver Dis 2008;28:386.

35. Francque S, Verrijken A, Mertens I, et al. Noncirrhotic human nonalcoholic fatty liver disease induces portal hypertension in relation to the histological degree of steatosis. Eur J Gastroenterol Hepatol 2010;22:1449.

36. Thomopoulos KC, Theocharis GJ, Tsamantas AC, et al. Liver steatosis is an independent risk factor for treatment failure in patients with chronic hepatitis C. Eur J Gastroenterol Hepatol 2005; 17:149.

37. Palmentieri B, de Sio I, La Mura V, et al. The role of bright liver echo pattern on ultrasound B-mode examination in the diagnosis of liver steatosis. Dig Liver Dis 2006;38:485. 
38. Saverymuttu SH, Joseph AE, Maxwell JD. Ultrasound scanning in the detection of hepatic fibrosis and steatosis. Br Med J (Clin Res Ed) 1986; 292:13.

39. Taylor KJ, Riely CA, Hammers L, et al. Quantitative US attenuation in normal liver and in patients with diffuse liver disease: importance of fat. Radiology 1986;160:65.
40. Kim SH, Lee JM, Kim JH, et al. Appropriateness of a donor liver with respect to macrosteatosis: application of artificial neural networks to US images-initial experience. Radiology 2005; 234:793.

41. Hricak H, Cruz C, Romanski R, et al. Renal parenchymal disease: sonographic-histologic correlation. Radiology 1982;144:141. 